\title{
Asymmetric chiral metamaterial circular polarizer based on four U-shaped split ring resonators
}

\author{
Mehmet Mutlu, ${ }^{1, *}$ Ahmet E. Akosman, ${ }^{1}$ Andriy E. Serebryannikov, ${ }^{2}$ and Ekmel Ozbay ${ }^{1}$ \\ ${ }^{1}$ Department of Electrical and Electronics Engineering, Nanotechnology Research Center, Bilkent University, 06800 Ankara, Turkey \\ ${ }^{2}$ Department of Electrical Engineering, E-3, Hamburg University of Technology, D-21071 Hamburg, Germany \\ *Corresponding author: mutlu@ee.bilkent.edu.tr
}

Received February 1, 2011; revised March 6, 2011; accepted March 28, 2011; posted March 31, 2011 (Doc. ID 141900); published April 28, 2011

\begin{abstract}
An asymmetric chiral metamaterial structure is constructed by using four double-layered U-shaped split ring resonators, which are each rotated by $90^{\circ}$ with respect to their neighbors. The peculiarity of the suggested design is that the sizes of the electrically and magnetically excited rings are different, which allows for equalizing the orthogonal components of the electric field at the output interface with a $90^{\circ}$ phase difference when the periodic structure is illuminated by an $x$-polarized wave. As a result, left-hand circular polarization and right-hand circular polarization are obtained in transmission at $5.1 \mathrm{GHz}$ and $6.4 \mathrm{GHz}$, respectively. The experiment results are in good agreement with the numerical results. (C) 2011 Optical Society of America

OCIS codes: $160.3918,160.1585,230.5440$
\end{abstract}

The chirality concept in metamaterials has attracted significant attention since Pendry predicted that a chiral route can be used in order to obtain negative refraction [1]. A chiral metamaterial (CMM) is not identical to its mirror image, i.e., it cannot be brought into congruence with its mirror image unless it is lifted off the substrate [2]. For such materials, at the resonance frequencies cross-coupling between electric and magnetic fields exists and, therefore, right-hand circularly polarized (RCP) and left-hand circularly polarized (LCP) waves encounter different transmission coefficients [3]. In other words, a CMM can lead to the rotation of the wave polarization [4]. Due to their interesting properties, e.g., giant optical activity and circular dichroism, CMMs can be important for optical applications [ $\underline{5}-10]$. For CMMs, the chirality parameter, $\kappa$, characterizes the strength of the crosscoupling between the magnetic and electric fields. Thus, the constitutive relations in a chiral medium are written as [11]

$$
\left(\begin{array}{l}
\mathbf{D} \\
\mathbf{B}
\end{array}\right)=\left(\begin{array}{cc}
\varepsilon_{0} \varepsilon & i \kappa / c_{0} \\
-i \kappa / c_{0} & \mu_{0} \mu
\end{array}\right)\left(\begin{array}{c}
\mathbf{E} \\
\mathbf{H}
\end{array}\right)
$$

In this Letter, we study the potential of an electrically thin, asymmetric chiral circular polarizer structure, which is composed of isotropic materials, in obtaining LCP and RCP waves using an $x$-polarized incident wave. Circular polarization is an important property for antenna applications [12-14], laser applications [15,16], remote sensors, and liquid crystal displays $[17,18]$. In the design process, we benefit from the metamaterial structure composed of two resonators, which are rotated by $90^{\circ}[\underline{6}]$ with respect to one another. The design proposed here is similar to those in Refs. [3, 19]; however, it is distinguished in that asymmetry is introduced by reducing the sizes of the electrically excited split ring resonators (SRRs) in order to modify the transmission at the $x$ polarization. By using this size reduction, the magnitudes of the transmitted electric fields in the $x$ and $y$ directions are equalized at the resonance frequencies, with a $90^{\circ}$ phase difference. The structure works as a left-hand and right-hand circular polarizer around the lower and higher resonance frequencies, respectively.

Circular polarization can be obtained from a linearly polarized incident wave by using a CMM, due to the different circular polarization transmission coefficients for LCP and RCP waves at the resonances. For instance, for the CMM structures given in Refs. [3,5,20], the transmission differences between RCP and $\overline{\mathrm{L}} \overline{\mathrm{C}} \overline{\mathrm{T}}$ waves are $8 \mathrm{~dB}$, $15-18 \mathrm{~dB}$, and $10 \mathrm{~dB}$, respectively. The structure proposed in this Letter aims to introduce a larger magnitude difference between LCP and RCP waves at the output interface to obtain circular polarization.

Figure 1 shows the geometry of the CMM unit cell used in the simulations and experiments. The unit cell consists of four double-layered U-shaped SRRs placed on both sides of an FR-4 board with a relative permittivity of 4 and a dielectric loss tangent of 0.025. Copper that is $30 \mu \mathrm{m}$ thick is used for the metallic parts. SRRs are positioned so that they are rotated by $90^{\circ}$ with respect to their neighbors.

We started the analysis with numerical simulations of the CMM structure using CST Microwave Studio (Computer Simulation Technology AG, Germany), which is a commercial software that is based on the finite integration method. The boundaries are selected to be periodic

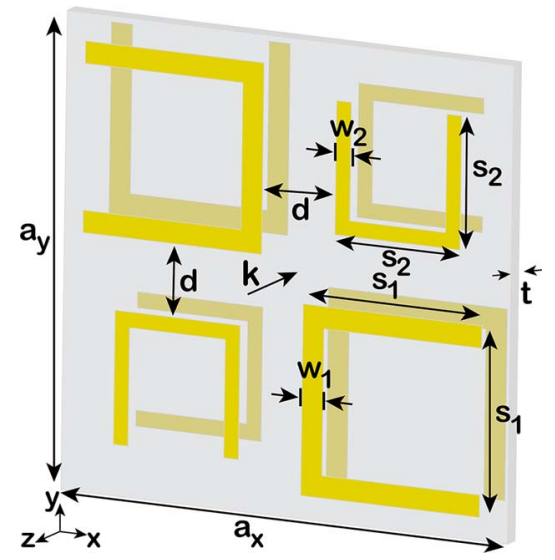

Fig. 1. (Color online) Geometry of the simulated and fabricated chiral metamaterial unit cell. 
in the $x$ and $y$ directions and open in the $z$ direction. The material is excited by a plane wave propagating in the direction of $\mathbf{k}(-z)$, as shown in Fig. 1 , with the electric field in the $x$ direction. The following geometric parameters are used in the simulations and experiments: $a x=a_{y}=15 \mathrm{~mm}, s_{1}=6 \mathrm{~mm}, s_{2}=4.2 \mathrm{~mm}, w_{1}=0.7 \mathrm{~mm}$, $w_{2}=0.5 \mathrm{~mm}, d=2.6 \mathrm{~mm}$, and $t=1.5 \mathrm{~mm}$. The structure is electrically thin since $t / \lambda$ is 0.024 and 0.03 for $5.1 \mathrm{GHz}$ and $6.4 \mathrm{GHz}$, respectively. In addition, the periods in the $x$ and $y$ directions are also electrically small, since $a x / \lambda$ is 0.255 at $5.1 \mathrm{GHz}$ and 0.32 at $6.4 \mathrm{GHz}$.

For the experiments, we fabricated the structure with the dimension of 15 by 15 unit cells. The experiment is conducted using two standard horn antennas facing each other at a $50 \mathrm{~cm}$ distance. The structure is placed in the middle between the antennas. The transmission coefficient measurements are performed using an HP-8510C network analyzer (Agilent Technologies, USA). In order to obtain the transmission characteristics of the structure, the $x$ and $y$ components of the transmitted field are studied in the simulations and experiments in terms of the transmission coefficients $T_{x x}$ and $T_{y x}$.

The ratio $\left|T_{y x}\right| /\left|T_{x x}\right|$ and the phase difference $\Phi\left(T_{y x}\right)-\Phi\left(T_{x x}\right)$ are presented in Figs. 2(a) and 2(b). The resonance peaks in Fig. 2(a) correspond to the frequencies where the electromagnetic coupling between the top and bottom layers is strong, so that $T_{y x}$ is large. According to the numerical results in Fig. 2(b), phase differences between $T_{y x}$ and $T_{x x}$ are (A) $89.8^{\circ}$ and (B) $-89.2^{\circ}$ at $5.1 \mathrm{GHz}$ and $6.4 \mathrm{GHz}$, respectively. In addition, at these frequencies, the ratios of the magnitudes of $T_{y x}$ and $T_{x x}$ are 1.03 and 0.994 , respectively. These results prove that the transmitted waves are LCP and $\mathrm{RCP}$ waves at $5.1 \mathrm{GHz}$ and $6.4 \mathrm{GHz}$, respectively. The experimental data shown in Fig. 2 is in good agreement with the numerical results.

Circular polarization transmission coefficients can be calculated from the linear transmission coefficients $T_{x x}$, $T_{y x}, T_{x y}$, and $T_{y y}[\underline{11}]$. Since the metamaterial structure

(a)

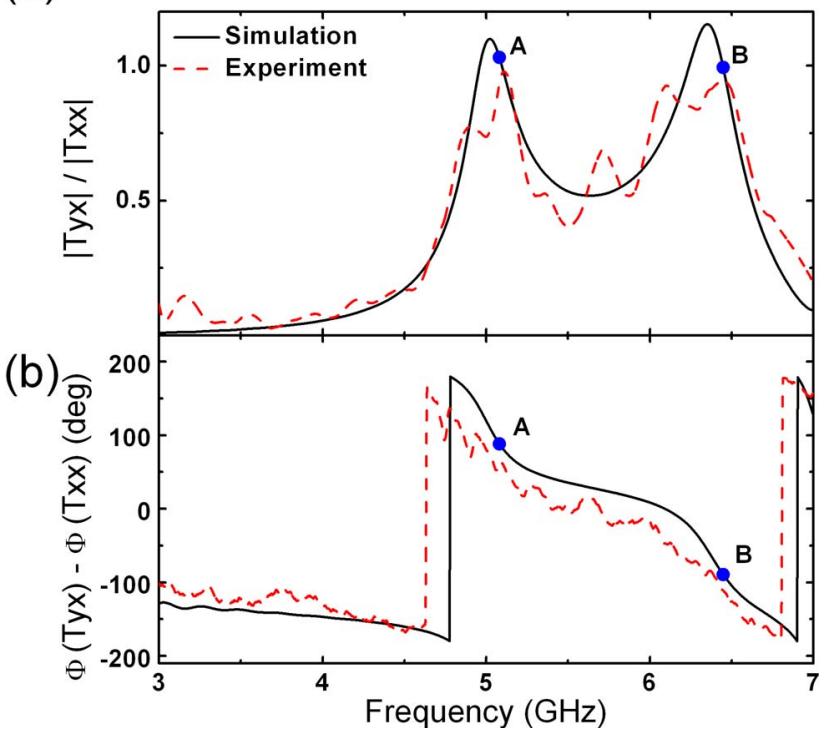

Fig. 2. (Color online) (a) Ratio of magnitudes of $T_{y x}$ and $T_{x x}$, and (b) phase difference between $T_{y x}$ and $T_{x x}$. lacks $C_{4}$ symmetry because SRRs of different sizes are used, the four circular transmission coefficients, $T_{++}$, $T_{-+}, T_{+-}$, and $T_{--}$should be calculated in order to completely characterize the response of the CMM. However, the structure provides circular polarization only if the incident wave is $x$-polarized. Therefore, $E_{y}$ is assumed to be zero. Under this assumption, we define the circular transformation coefficients $C^{+}(\mathrm{RCP})$ and $C^{-}(\mathrm{LCP})$. The conversion from the linear transmission coefficients to circular transformation coefficients becomes, simply, $C^{ \pm}=T_{x x} \pm i T_{y x}$ [3]. It is noteworthy that the calculated transformation coefficients are only valid for an $x$-polarized incident field, since the structure lacks $C_{4}$ symmetry.

Numerical and experimental circular transformation coefficients for the LCP and RCP components are presented in Fig. 3. In this context, these parameters are defined as the magnitude of the RCP and LCP waves at the output interface due to an $x$-polarized incident field. In Fig. 3, $0 \mathrm{~dB}$ corresponds to the magnitude of the incident $x$-polarized electric field. The minimum contribution of the RCP component is observed at $5.1 \mathrm{GHz}$ as $-37 \mathrm{~dB}$ in Fig. 3(a) and, similarly, the minimum for the LCP component is obtained at $6.4 \mathrm{GHz}$ as $-43 \mathrm{~dB}$. The experimental results provided in Fig. 3(b) are in good agreement with the numerical results.

It follows from the presented results that the circular polarizer feature of the studied CMM originates from the different circular polarization transformation coefficients for the RCP and LCP components, due to an $x$ polarized incident wave. One of the circular polarizations is eliminated at the resonance frequencies, whereas the other polarization is transmitted with a small loss. As a result, at $5.1 \mathrm{GHz}$, the transmitted wave is LCP. Similarly, at $6.4 \mathrm{GHz}$, the RCP wave is transmitted.

As mentioned above, the proposed structure is similar to the structure studied in Ref. [3]. In this work, the sizes of all the SRRs are equal (geometric parameters given as $s_{1}$ and $w_{1}$ ), which means that at the frequencies of

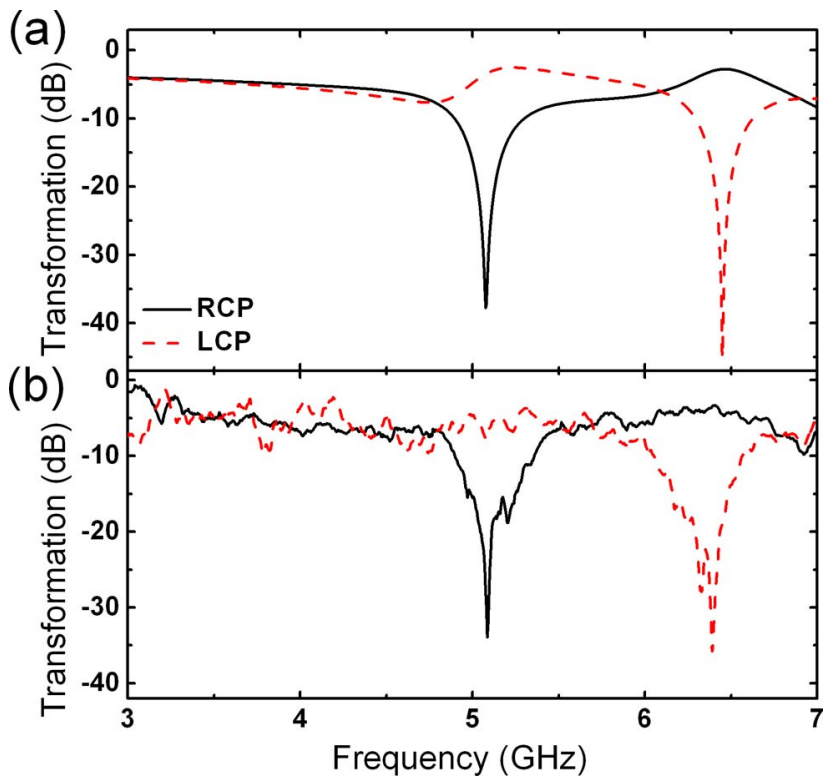

Fig. 3. (Color online) (a) Numerical and (b) experimental results for the circular transformation coefficients. 


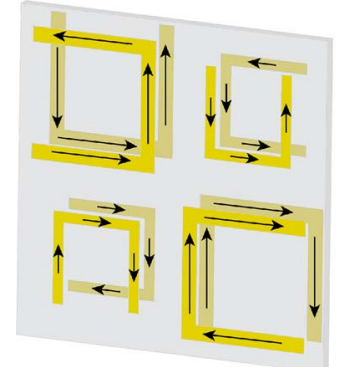

$5.1 \mathrm{GHz}$

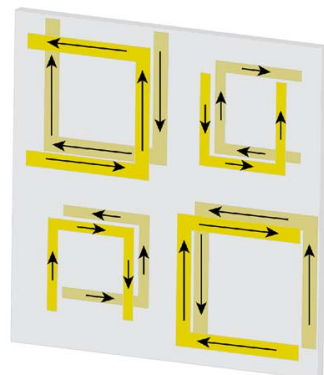

$6.4 \mathrm{GHz}$
Fig. 4. (Color online) Directions of the surface currents on the SRRs due to the incident electric field polarized in the $x$ direction, at $5.1 \mathrm{GHz}$ and $6.4 \mathrm{GHz}$

minimum RCP and LCP wave transmission, all the SRRs are in the vicinity of resonance. As a result, $T_{x x}$ is around $-20 \mathrm{~dB}$ near the resonance frequencies, while $T_{y x}$ is obtained to be $-6 \mathrm{~dB}$ at those frequencies. Hence, since $T_{x x}$ and $T_{y x}$ are significantly different, the resulting polarization is elliptical.

In the proposed design, the sizes of the SRR pairs, which are shown in Fig. 1, are reduced in order to increase $T_{x x}$. Numerical results reveal that, in this case, $T_{x x}$ is increased (to approximately $-6.5 \mathrm{~dB}$ around the resonance frequencies) by using the effect exerted by the smaller SRR pairs being out of resonance. In order to understand the nature of the resonances occurring at $5.1 \mathrm{GHz}$ and $6.4 \mathrm{GHz}$, the surface current distribution is studied numerically. The directions of the induced surface currents at the two resonances are shown in Fig. 4. Smaller arrows are used for the smaller SRR pairs in order to indicate that the magnitude of the currents on those SRRs are smaller when compared to the large SRR pairs as an effect of being out of resonance. As a result, transmission of the $x$-polarization, $T_{x x}$, is increased, and the geometrical parameters are carefully optimized such that at the frequencies where $\mid \Phi\left(T_{y x}\right)-$ $\Phi\left(T_{x x}\right)\left|=90^{\circ}, \quad\right| T_{y x}|/| T_{x x} \mid$ is approximately equal to unity. According to the simulations performed for a $y$ polarized incident field, the transmitted wave is elliptical, with $\left|T_{x y}\right| /\left|T_{y y}\right|=0.44$ and $\Phi\left(T_{x y}\right)-\Phi\left(T_{y y}\right)=105^{\circ}$ at $5.1 \mathrm{GHz}$. At $6.4 \mathrm{GHz}, \quad\left|T_{x y}\right| /\left|T_{y y}\right|=0.39$ and $\Phi\left(T_{x y}\right)-$ $\Phi\left(T_{y y}\right)=5^{\circ}$, and these values do not correspond to circular polarization.

For the mutually $90^{\circ}$ rotated SRR pairs, the resonance levels are determined in line with the longitudinal magnetic dipole to magnetic dipole coupling [9]. Numerical study shows that at the lower resonance frequency, the surface currents on the twisted SRR pairs are in the same direction, leading to parallel magnetic dipole moments for the twisted pairs. In contrast, at the higher resonance frequency, the surface currents on the twisted SRR pairs are antiparallel, which results in antiparallel magnetic dipole moments.

The major advantage of the structure is being electrically very thin. Another advantage is that the structure is planar and easy to fabricate. On the other hand, narrowband operating frequency can be a drawback, depending on the application. However, broadband response may be achieved by extending the design to three dimensions.
To summarize, we have designed an asymmetric CMM structure based on four double-layered U-shaped SRRs. LCP and RCP waves are obtained in the vicinity of $5.1 \mathrm{GHz}$ and $6.4 \mathrm{GHz}$, respectively, when the structure is illuminated by an $x$-polarized wave propagating in the $-z$ direction. The design can be utilized as a circular polarizer for microwave applications. As a future work, the ideas of the suggested design can be adapted for terahertz and optical applications.

This work is supported by the European Union (EU) under projects PHOME, ECONAM, and N4E; by The Scientific and Technological Research Council of Turkey (TUBITAK) under projects 109E301, 107A004, and 107A012; and the State Planning Organization (DPT) under project DPT-HAMIT. One of the authors (E. Ozbay) also acknowledges partial support from the Turkish Academy of Sciences.

\section{References}

1. J. B. Pendry, Science 306, 1353 (2004).

2. M. Decker, M. W. Klein, M. Wegener, and S. Linden, Opt. Lett. 32, 856 (2007).

3. Z. Li, R. Zhao, T. Koschny, M. Kafesaki, K. B. Alici, E. Colak, H. Caglayan, E. Ozbay, and C. M. Soukoulis, Appl. Phys. Lett. 97, 081901 (2010).

4. A. Demetriadou and J. B. Pendry, J. Phys. Condens. Matter 21, 376003 (2009).

5. S. Zhang, Y. S. Park, J. Li, X. Lu, W. Zhang, and X. Zhang, Phys. Rev. Lett. 102, 023901 (2009).

6. H. Liu, D. A. Genov, D. M. Wu, Y. M. Liu, Z. W. Liu, C. Sun, S. N. Zhu, and X. Zhang, Phys. Rev. B 76, 073101 (2007).

7. T. Q. Li, H. Liu, T. Li, S. M. Wang, F. M. Wang, R. X. Wu, P. Chen, S. N. Zhu, and X. Zhang, Appl. Phys. Lett. 92, 131111 (2008).

8. H. Liu, J. X. Cao, S. N. Zhu, N. Liu, R. Ameling, and H. Giessen, Phys. Rev. B. 81, 241403 (2010).

9. N. Liu, H. Liu, S. Zhu, and H. Giessen, Nat. Photon. 3, 157 (2009).

10. A. V. Rogacheva, V. A. Fedotov, A. S. Schwanecke, and N. I. Zheludev, Phys. Rev. Lett. 97, 177401 (2006).

11. R. Zhao, T. Koschny, and C. M. Soukoulis, Opt. Express 18, 14553 (2010).

12. Nasimuddin, X. Qing, and Z. N. Chen, IEEE Trans. Antenna Propagat. 59, 285 (2011)

13. K. L. Wong, C. C. Huang, and W. S. Chen, IEEE Trans. Antenna Propagat. 50, 75 (2002).

14. J. Y. Sze, K. L. Wong, and C. C. Huang, IEEE Trans. Antenna Propagat. 51, 2141 (2003).

15. T. V. Liseikina and A. Macchi, Appl. Phys. Lett. 91, 171502 (2007).

16. A. H. Nejadmalayeri and P. R. Herman, Opt. Lett. 31, 2987 (2006).

17. Z. Ge, M. Jiao, R. Lu, T. X. Wu, S. T. Wu, W. Y. Li, and C. K. Wei, J. Display Technol. 4, 129 (2008).

18. Q. Hong, T. Wu, X. Zhu, R. Lu, and S. T. Wu, Opt. Express 13, 8318 (2005).

19. X. Xiong, W. H. Sun, Y. J. Bao, M. Wang, R. W. Peng, C. Sun, X. Lu, J. Shao, Z. F. Li, and N. B. Ming, Phys. Rev. B 81, 075119 (2010).

20. J. Zhou, J. Dong, B. Wang, T. Koschny, M. Kafesaki, and C. M. Soukoulis, Phys. Rev. B 79, 121104 (2009). 